\title{
Is a policy of watch and wait after a complete response following neoadjuvant treatment for locally advanced rectal adenocarcinoma justified? Should we reset the limit?
}

\author{
Carlo Eugenio Vitelli • Francesco Stipa • \\ Ugo De Paula
}

Received: 8 November 2013/Accepted: 13 November 2013/Published online: 28 November 2013

(C) Springer-Verlag Italia 2013

\begin{abstract}
Keywords Neoadjuvant treatment $\cdot$ Rectal carcinoma Complete response to chemoradiation therapy $\cdot$ Conservative treatment of rectal carcinoma . Evaluation of neoadjuvant treatment
\end{abstract}

Radical surgery has been considered, in the past century, the unique and more effective therapeutic option for locally advanced rectal adenocarcinoma. Standardization of surgical technique [1] has reduced the local recurrence rates to one digit percentages, and the addition of radiation therapy treatments combined with less toxic chemotherapy regimens either pre- or post-operatively has significantly improved the short- and long-term outcomes of these patients. The systematic use of neoadjuvant therapy has led to the common observation that at least $20-30 \%$ of patients (depending on the CHT regimen used and the timing of surgery) are completely free of tumor (CR: ypT0ypN0) in the resected specimens [2,3].

Despite these encouraging results, we continue to operate on these patients with a low-mortality rate but with a significant percentage of functional sequelae (low anterior resection syndrome, leaks, stenosis, sexual and urinary

\section{E. Vitelli $(\bowtie) \cdot$ F. Stipa}

Department of Surgery, Azienda Ospedaliera San Giovanni Addolorata-Roma, Via dell'Amba Aradam 9, 00184 Rome, Italy e-mail: cevitelli@me.com

F. Stipa

e-mail: stipafrancesco@hsangiovanni.roma.it

\section{U. De Paula}

Department of Radiation Oncology, Azienda Ospedaliera San Giovanni Addolorata-Roma, Via dell'Amba Aradam 9, 00184 Rome, Italy

e-mail: depaulaugo@hsangiovanni.roma.it problems, need for a diverting stoma). These complications occur more frequently than usually reported in the surgical literature, especially when the postoperative evaluation is performed by a Registered Nurse or a surgeon not involved with the operation [4].

If we all agree that small $\mathrm{T} 1$ lesions without particularly aggressive features can be safely treated by wide local excision (either transanal or better via TEM, Transanal Endoscopic Microsurgery) [5] why should we modify our thinking and treat the patients who have reached a complete response after the preoperative treatment in a different way?

Pioneristic work [6] has demonstrated that in patients who respond to the neoadjuvant treatment, a policy of intense observation without surgery is possible and not followed by an increase in cancer related mortality. The main difficulty in accepting this "obvious" policy, by the surgical community, has been that, so far, no test has shown to be $100 \%$ accurate in identifying a complete response to the preoperative treatment, thus in discriminating which patient to observe from which patient to operate [7].

Another important issue that has recently emerged is that the arbitrarily chosen 6-week waiting period (after the completion of the neoadjuvant treatment and the operation), if prolonged up to 8 or 12 weeks brings a consistent increase in the percentage of complete responders up to $30 \%$ and above [8]. This observation can be explained by the prolonged effect of the radiation therapy subministered to the tumor bed.

At our institution we have developed a prospective observational protocol, IRB approved, for patients with locally advanced non-metastatic extraperitoneal rectal adenocarcinomas consisting of re-evaluation at 6 weeks after the end of ERT and then at 12 weeks for patients 
showing an initial significant clinical response at the first evaluation. In our protocol, the patients are studied with a baseline CT scan, MRI, PET, and EUS and then re-evaluated by proctoscopy and PE at 6 weeks after the completion of the ERT and CHT neoadjuvant treatments. If they have had a poor response, they go to immediate radical surgery. If the response has been significant, the patients wait six more weeks, at which time the MRI, EUS, and PET scans are repeated and compared. Before surgery, a proctoscopy with tattooing of the area is performed, and endoscopic biopsies are done. Complete responders by radiological, clinical, and endoscopic criteria undergo a full-thickness excision performed via TEM. If no cancer cells are seen on the TEM specimen, the patient is allocated to the observation arm, whereas if cancer is still present, the patient undergoes radical anterior resection with a protective stoma. In both cases, the patient is then referred to the medical oncologist to complete the scheduled chemotherapy regimen.

The case open for discussion remains when there is a significant but not complete response (ypT1) on the TEM specimen: should we include to the conservative treatment arm these patients too? It is possible that simply by extending the observation period after the completion of treatment to 18 rather than 12 weeks will increase the percentage of complete responders?

As surgical oncologists we should feel compelled to answer these questions by entering eligible patients in this type of prospective studies because our feeling is that the surgical treatment of rectal carcinoma can be tailored to the single patient with much less extensive procedures and far less morbidity. Furthermore, in a period of cost containment, such as the one we are witnessing currently, a transanal procedure rather than a transabdominal procedure carries a much lower economic burden for the society and a faster recovery for the patient.
Conflict of interest None.

\section{References}

1. Heald RJ (1988) The 'Holy Plane' of rectal surgery. J Royal Soc Med 81:503-508

2. Valentini V, Coco C, Picciocchi A, Morganti AG, Trodella L, Ciabattoni A, Cellini F, Barbaro B, Cogliandolo S, Nuzzo G, Doglietto GB, Ambesi-Impiombato F, Cosimelli M (2002) Does downstaging predict improved outcome after preoperative chemoradiation for extraperitoneal locally advanced rectal cancer? A long-term analysis of 165 patients. Int J Rad Oncol Biol Phys 53(3):664-674

3. Haas M, Nelemans PJ, Valentini V, Das P, Rödel C, Kuo LJ, Calvo FA, García-Aguilar J, Glynne-Jones R, Haustermans K, Mohiuddin M, Pucciarelli S, Small W Jr, Suárez J, Theodoropoulos G, Biondo S, Beets-Tan RG, Beets GL (2010) Long-term outcome in patients with a pathological complete response after chemoradiation for rectal cancer: a pooled analysis of individual patient data. Lancet Oncol 11(9):835-844

4. Bruner DW, Calvano T (2007) The sexual impact of cancer and cancer treatments in men. Nurs Clin N Am 42:555-580

5. Bökkerink GMJ et al (2011) The CARTS study: chemoradiation therapy for rectal cancer in the distal rectum followed by organsparing transanal endoscopic microsurgery. BMC Surg 11:34-38

6. Habr-Gama A, Perez RO, Nadalin W, Sabbaga J, Ribeiro U Jr, Silva e Sousa AH Jr, Campos FG, Kiss DR, Gama-Rodrigues J (2004) Operative versus nonoperative treatment for stage 0 distal rectal cancer following chemoradiation therapy: long-term results. Ann Surg 240(4):711-717

7. Augestad KM, Lindsetmo RO, Stulberg J, Reynolds H, Senagore A, Champagne B, Heriot AG, Leblanc F, Delaney CP; International Rectal Cancer Study Group (IRCSG) (2010) International preoperative rectal cancer management: staging, neoadjuvant treatment, and impact of multidisciplinary teams. World J Surg 34:2689-2700

8. Garcia-Aguilar J, Smith DD, Avila K, Bergsland EK, Chu P, Krieg RM; Timing of Rectal Cancer Response to Chemoradiation Consortium (2011) Optimal timing of surgery after chemoradiation for advanced rectal cancer: preliminary results of a multicenter, nonrandomized phase II prospective trial. Ann Surg 254:97-102 